\title{
Upconversion of Broadband Infrared Radiation into Visible Light for Different Pumping Parameters
}

\author{
Man Nen Litvinova, ${ }^{1}$ Victor Krishtop, ${ }^{1,2}$ Evgeniy Tolstov, ${ }^{1}$ Vladimir Troilin, ${ }^{1}$ \\ Larisa Alekseeva, ${ }^{1}$ and Veronika Litvinova ${ }^{1}$ \\ ${ }^{1}$ Physics Department, Far Eastern State Transport University, 47 Seryshev Street, Khabarovsk 680021, Russia \\ ${ }^{2}$ Electrophysics Department, Kwangwoon University, 20 Kwangwoon Road, Nowon-Gu, Seoul 139-701, Republic of Korea \\ Correspondence should be addressed to Victor Krishtop; krishtop@list.ru
}

Received 16 December 2012; Accepted 3 February 2013

Academic Editor: Paola Luches

Copyright (C) 2013 Man Nen Litvinova et al. This is an open access article distributed under the Creative Commons Attribution License, which permits unrestricted use, distribution, and reproduction in any medium, provided the original work is properly cited.

The influence of pump radiation parameters such as the polarization and the spectral width of infrared radiation on the conversion of broadband radiation in lithium niobate crystals was investigated. The spectra of converted radiation were calculated for two types of phase matching in the negative uniaxial crystal by taking into account the convergence of the light beam in the crystal. Experimental spectra were obtained and compared with the calculated spectra.

\section{Introduction}

Systems based on thermographic phosphors, digital holograms, and other conversion mechanisms have been widely used to achieve thermal-to-visible conversion $[1,2]$. Optical methods for the conversion of broadband infrared radiation are based on frequency-mixing processes such as second harmonic generation and sum frequencies generation in nonlinear optical crystals and are used for the visualization of infrared images of thermal objects $[2,3]$ and in other applications [4]. These methods appear to offer the most advantage for the analysis of two-dimensional (and three-dimensional) infrared images. The important advantage of optical methods for the visualization of infrared radiation in nonlinear optical crystals, unlike electrooptical transformation methods, is that they preserve the phase information and consequently enable further coherent image processing (e.g., holography in the infrared range with no inertial visualization).

Since the late 1960s, studies have focused on problems involved in the conversion of broadband infrared radiation into the visible spectral range by nonlinear optics methods [5]. First, the conversion of broadband infrared radiation in nonlinear crystals was carried out by using narrowband laser pump radiation. However, this method for the visualization of infrared images has some disadvantages such as the spotty image structure caused by the coherence of laser pump radiation, distortion of the image size along one of the directions (in the plane of phase matching), narrow spectral frequency interval of the converted radiation of $20-30 \mathrm{~cm}^{-1}$ (occasionally, of $200-300 \mathrm{~cm}^{-1}$ ), and sharp deterioration of the converted image when the spectral frequency interval of the infrared radiation is increased or when the pump radiation is focused [6].

At the same time, there are a number of interesting experimental and theoretical results of studies of the conversion of broadband infrared radiation by the use of incoherent pump radiation. It has been shown that when certain conditions are fulfilled, the process of harmonic generation for incoherent pump radiation can be more effective than that for coherent laser pump radiation $[6,7]$. Then, nonlinear visualization of the infrared image of a thermal object by using pumping from nonlaser sources is the most reasonable method.

Nonlinear visualization of infrared radiation is realized using conversion systems for infrared radiation. The characteristics of conversion systems for infrared images in nonlinear crystals with pumping from incoherent radiation sources have been reported [8]. 
The influence of thermal object temperature and pump radiation parameters on the spectrum and conversion efficiency of broadband infrared radiation has been investigated [8-10]. In these studies, the spectra of converted infrared radiation were calculated for collinear light beams. However, in the case of convergent light beams, it is necessary to take into account the noncollinear light wave interactions, which essentially increase the conversion efficiency of broadband infrared radiation in nonlinear crystals [11].

This study investigates the influence of pump radiation parameters such as the polarization and spectral width of infrared radiation on the conversion of broadband infrared radiation in nonlinear optical crystals. The spectra of converted broadband infrared radiation in nonlinear optical crystals were calculated by taking into account the noncollinear interactions of light waves [12]. Approximate calculations were carried out when the power of a generated wave is so small that it is possible to consider the amplitudes of two propagating waves to have been constant through the entire length of a crystal. The calculated spectra were compared to the corresponding experimental spectra. The experimental spectra were obtained for nonlinear negative crystals of lithium niobate (LN).

\section{Model}

Sum-frequency generation and second harmonic generation occur when the propagation direction of a beam of broadband infrared radiation coincides with the phase matching direction for one of the input frequencies. The waves of frequencies $\omega_{1}=\omega_{3} / 2-\Omega$ and $\omega_{2}=\omega_{3} / 2+\Omega$ contribute to a generated wave of frequency $\omega_{3}$, where $\Omega$ is the interval between frequencies $\omega_{1}$ and $\omega_{2}$ and frequency $\omega_{3} / 2$ [13]. It is assumed that the amplitudes of two propagating waves are constant throughout the entire length of a crystal.

The intensity of the converted radiation of frequency $\omega_{3}$ is given as

$$
I_{3}(\Omega)=A I_{1}\left(\omega_{1}\right) I_{2}\left(\omega_{2}\right) \frac{\sin ^{2}(\Delta k l / 2)}{(\Delta k / 2)^{2}}
$$

where $A$ is a constant proportional to the squared component of the nonlinear susceptibility tensor, $I_{1}\left(\omega_{1}\right)$ and $I_{2}\left(\omega_{2}\right)$ are the intensities of interacting light waves, $l$ is the length of a crystal along the propagation directions of waves, and $\Delta k$ is the disturbance of phase matching. The disturbance of phase matching is given as [8-13]

$$
\begin{aligned}
\Delta k & =k_{3}-\left(k_{1}+k_{2}\right) \\
& =\frac{1}{c}\left[\frac{\omega_{3}}{2}\left(n_{1}+n_{2}-2 n_{3}\right)+\Omega\left(n_{1}-n_{2}\right)\right],
\end{aligned}
$$

where $k_{1}\left(\omega_{1}\right), k_{2}\left(\omega_{2}\right)$, and $k_{3}\left(\omega_{3}\right)$ are wave numbers of the three waves as they travel through the medium and $n_{1}\left(\omega_{1}\right)$, $n_{2}\left(\omega_{2}\right)$, and $n_{3}\left(\omega_{3}\right)$ are refractive indexes that depend on the polarization and direction of the waves that pass through the crystal. The values of the refractive indexes were calculated by the Sellmeier equations.
The intensity of the converted broadband radiation with frequency $\omega_{3}$ is provided by the resulting interaction of waves of all frequencies $\omega_{1}$ and $\omega_{2}$ that are equidistant from $\omega_{3} / 2$. The spectral intensity $I\left(\omega_{3}\right)$ for a collinear light beam is given as [8-10]

$$
I_{3}\left(\omega_{3}\right)=A \int_{0}^{\omega_{3} / 2} I_{1}\left(\omega_{1}\right) I_{2}\left(\omega_{2}\right) \frac{\sin ^{2}(\Delta k l / 2)}{(\Delta k / 2)^{2}} d \Omega .
$$

The intensity of the converted broadband radiation taking into account the interactions of noncollinear light beams along a direction $\theta^{\prime}$ is given as

$$
I\left(\omega_{3}, \theta^{\prime}\right)=A \int_{0}^{\alpha} \int_{0}^{\omega_{3} / 2} I_{1}\left(\omega_{1}\right) I_{2}\left(\omega_{2}\right) \frac{\sin ^{2}(\Delta k l / 2)}{(\Delta k / 2)^{2}} d \Omega d \alpha^{\prime},
$$

where $2 \alpha$ is the angle of convergence of an input beam in the crystal. The disturbance of phase matching $\Delta k$ depends on the refractive indexes $n_{1}\left(\omega_{1}\right), n_{2}\left(\omega_{2}\right)$, and $n_{3}\left(\omega_{3}\right)$, which for the first type of phase matching (oo-e) are defined by the expressions:

$$
\begin{gathered}
n_{1}\left(\omega_{1}\right)=n_{o}\left(\frac{\omega_{3}}{2}-\Omega\right) \\
n_{2}\left(\omega_{2}\right)=n_{o}\left(\frac{\omega_{3}}{2}+\Omega\right) \\
n_{3}\left(\omega_{3}, \theta^{\prime}+\alpha^{\prime}\right) \\
=\frac{n_{o}\left(\omega_{3}\right) n_{e}\left(\omega_{3}\right)}{\sqrt{n_{e}^{2}\left(\omega_{3}\right) \cos ^{2}\left(\theta^{\prime}+\alpha^{\prime}\right)+n_{o}^{2}\left(\omega_{3}\right) \sin ^{2}\left(\theta^{\prime}+\alpha^{\prime}\right)}}
\end{gathered}
$$

where $n_{o}$, and $n_{e}$ are refractive indexes for ordinary and extraordinary waves at the respective frequencies.

The intensity of the converted broadband radiation at the crystal output was calculated by taking into account the interactions of noncollinear light beams along all possible directions within the angle range of an input convergent beam of infrared broadband radiation [9-12]:

$$
\begin{aligned}
I_{3}\left(\omega_{3}\right)=A \int_{\theta-\alpha}^{\theta+\alpha} \int_{0}^{\alpha} \int_{0}^{\omega_{3} / 2} I_{1}\left(\omega_{1}\right) I_{2}\left(\omega_{2}\right) \\
\quad \times \frac{\sin ^{2}(\Delta k l / 2)}{(\Delta k / 2)^{2}} d \Omega d \alpha^{\prime} d \theta^{\prime}
\end{aligned}
$$

where $\theta$ is the angle of phase matching for the frequency $\omega_{3} / 2$ with respect to the optical axis of a crystal.

\section{Calculation}

Let us consider an algorithm for the calculation of the spectrum of broadband radiation converted in a nonlinear crystal.

The spectra of the converted broadband infrared radiation were calculated for two types of phase matching, which are usually the most suitable in nonlinear negative 
uniaxial crystals. In the first type of phase matching (oo-e), the input waves of angular frequencies $\omega_{1}$ and $\omega_{2}$ have ordinary polarization with respect to the crystal, but the generated wave of frequency $\omega_{3}$ has extraordinary polarization. In the second type of phase matching (oe-e), the input waves of angular frequencies $\omega_{1}$ and $\omega_{2}$ have orthogonal polarizations, but the generated wave of frequency $\omega_{3}$ has extraordinary polarization.

The intensity of the converted broadband radiation at the crystal output defines the resulting spectrum of converted radiation. First, the dependence of the intensity of the converted radiation $I(\Omega)$ at frequency $\omega_{3}$ on the frequency interval $\Omega$ between frequencies $\omega_{1}$ and $\omega_{2}$ and frequency $\omega_{3} / 2$ of the input broadband infrared radiation was calculated by (1). It was assumed that the waves at frequencies $\omega_{1}$ and $\omega_{2}$ were propagated in the direction of phase matching for one of the input frequencies $\omega_{0}$ (wavelength: $\lambda_{0}$ ). The calculation results showed that the intensity of the converted radiation $I(\Omega)$ quickly decreased for frequencies of the converted radiation that are smaller than $2 \omega_{0}$. The intensity of the converted radiation $I(\Omega)$ for frequencies of the converted radiation that are greater than $2 \omega_{0}$ depends on the frequency interval $\Omega$ between frequencies $\omega_{1}$ and $\omega_{2}$.

During the second harmonic generation, each frequency $\omega_{3}\left(\lambda_{3}\right)$ of converted infrared radiation can be obtained by various combinations of frequencies of the input radiation, as $\omega_{3}=\left(\omega_{3} / 2-\Omega\right)+\left(\omega_{3} / 2+\Omega\right)$. However, only one combination of frequencies $\omega_{1}\left(\lambda_{1}\right)$ and $\omega_{2}\left(\lambda_{2}\right)$ is possible for each value of frequency $\omega_{3}\left(\lambda_{3}\right)$ in the given phase matching direction.

Next, the intensity $I\left(\omega_{3}\right)$ of the converted radiation for the collinear interactions of light waves was calculated by (3). The spectrum of the converted radiation for the collinear interactions of light waves is unsymmetrical in relation to frequency $2 \omega_{0}$, corresponding to the maximum intensity. For frequencies $\omega_{3}>2 \omega_{0}$, the intensity of the converted radiation rapidly decreases. This is caused by reduction of the frequency interval $\Omega$ of interacting waves and by a decrease in the efficiency of the conversion of broadband infrared radiation. For frequencies $\omega_{3}<2 \omega_{0}$, converted radiation does not occur.

Finally, the intensity of the converted broadband radiation at the crystal output was calculated by (3) by taking into account the noncollinear interactions of light waves. The calculated spectra for a LN crystal (thickness: $11 \mathrm{~mm}$ ) are shown in Figure 1(a).

The spectra of the converted broadband radiation were calculated for the first type of phase matching (oo-e) when the axis of the convergence beam of broadband infrared radiation coincided with the phase matching direction for the wavelength $1.06 \mu$. The angle $\theta$ between the axis of the incident beam and the optical axis of the $\mathrm{LN}$ is $84.8^{\circ}$. In addition, it is assumed that the spectral range of infrared radiation is $0.67-1.7 \mu$.

The spectra of the converted broadband radiation were obtained for several values of the angle $2 \alpha$ of convergence of an input beam. The calculation results of the spectra of the converted broadband radiation showed that the increase in the convergence angle of an input beam results in the extension of the spectrum of converted radiation in the region of longer wavelengths and that the conversion efficiency of broadband infrared radiation increases [13].

In the general case, the shape of the spectrum depends on the location of the phase matching frequency in the input infrared radiation spectrum and on the propagation direction of infrared radiation with respect to the optical axis of the nonlinear crystal. The width of the spectrum of the converted radiation significantly depends on the type of nonlinear crystal, its thickness, and the type of interaction of light waves. The width of the converted radiation spectrum achieves its maximum when the phase matching frequency is chosen precisely in the middle of the frequency range of input infrared radiation. The increase in the spectral range of the input infrared radiation into the region of short wavelengths leads to the extension of the spectrum of converted radiation in the region of shorter wavelengths, and the conversion efficiency of broadband infrared radiation increases [13].

In addition, the spectra of the converted broadband radiation were calculated for the second type of phase matching $(o e-e)$. It is assumed that the angle $\theta$ between the axis of the incident beam and the optical axis of the crystal of LN is $84.8^{\circ}$ (phase matching direction for the wavelength $1.06 \mu$ ), the spectral range of infrared radiation is $0.67-1.7 \mu$, and the convergence of the incident beam is $5^{\circ}$. The calculated spectra for LN are shown in Figure 1(b).

When the input waves have orthogonal polarizations, two combinations are possible: $o\left(\lambda_{1}\right) e\left(\lambda_{2}\right)$ and $o\left(\lambda_{2}\right) e\left(\lambda_{1}\right)$. Therefore, the resulting spectrum of converted radiation for the oe-e type of phase matching has two maxima, which correspond to two cases of interaction $o\left(\lambda_{2}\right) e\left(\lambda_{1}\right)-e\left(\lambda_{3}\right)$ and $o\left(\lambda_{1}\right) e\left(\lambda_{2}\right)-e\left(\lambda_{3}\right)$.

The calculated spectra of the converted broadband radiation were compared to the corresponding experimental spectra obtained for LN.

\section{Experimental Data and Discussion}

The experimental spectra of the converted broadband radiation (Figure 2) were obtained by means of an experimental setup [8]. A lamp with a small tungsten filament is used as a source of infrared radiation.

The spectra of the broadband radiation converted in $\mathrm{LN}$ are shown in Figure 2(a) for two types of phase matching, with the spectral range of the input infrared radiation being $0.67-1.7 \mu$ and the convergence of the incident beam being $5^{\circ}$. The axis of an input beam of infrared radiation was directed at an angle of under $90^{\circ}$ to the optical axis of LN.

The spectrum of the converted broadband radiation for the oo-e type of phase matching has one maximum $(\lambda=0.53 \mu)$. The maximum of the spectrum of converted broadband radiation is shifted to a spectral range of longer wavelengths when the value of the phase matching angle $\theta_{c}$ is decreased. In contrast, it is shifted to a spectral range of shorter wavelengths when the value of the phase matching angle $\theta_{c}$ is increased. Furthermore, the spectrum maximum can be shifted by focusing the input beam. It is shifted to 


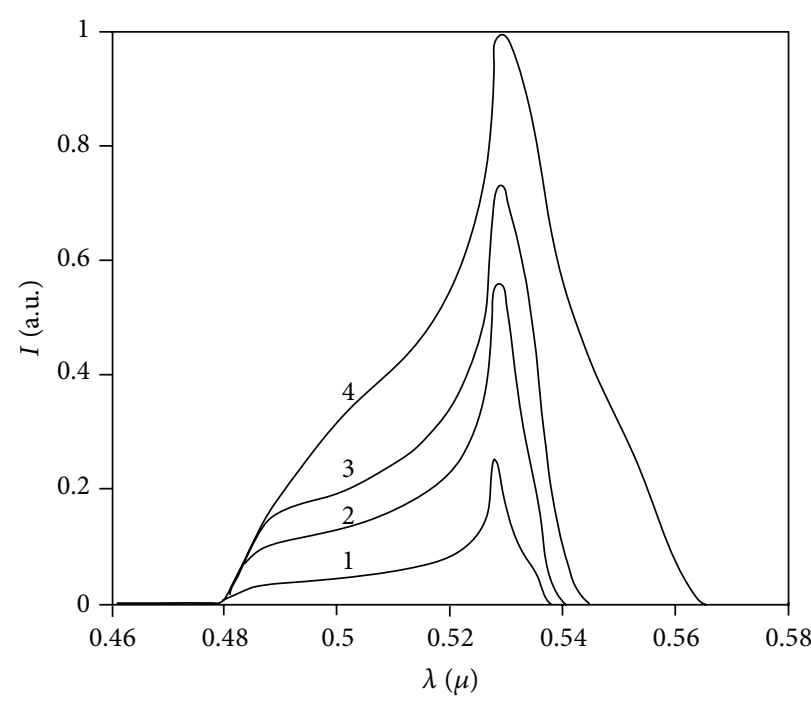

(a)

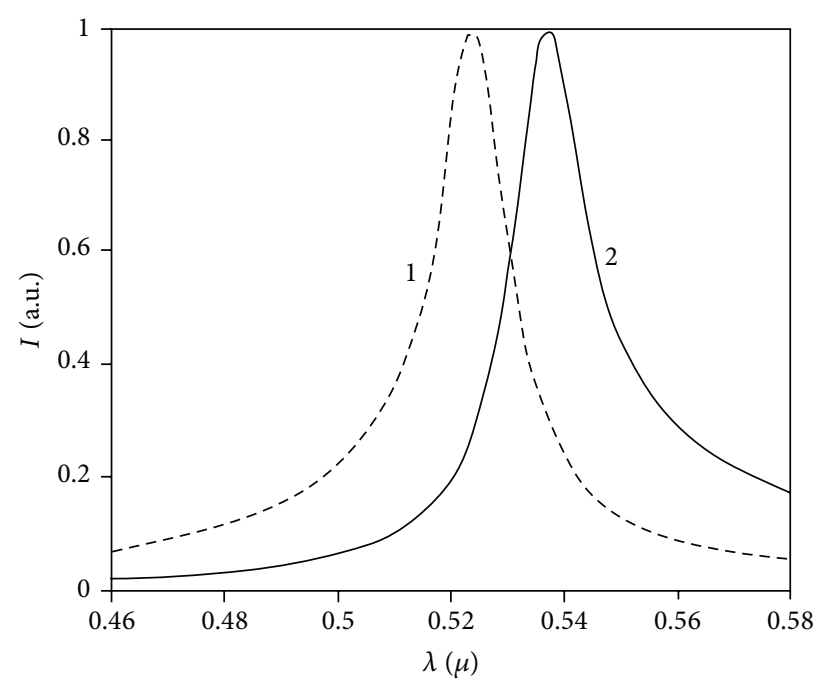

(b)

FIGURE 1: Calculated spectra of converted broadband radiation calculated for LN: (a) type of phase matching, oo-e; angle of convergence of input beam $2 \alpha: 1-1.2^{\circ} ; 2-3.4^{\circ} ; 3-7.6^{\circ} ; 4-10^{\circ}$ and (b) type of phase matching oe-e : $1-o\left(\lambda_{1}\right) e\left(\lambda_{2}\right)-e\left(\lambda_{3}\right) ; 2-o\left(\lambda_{2}\right) e\left(\lambda_{1}\right)-e\left(\lambda_{3}\right) ; \lambda_{1}>\lambda_{2}$.

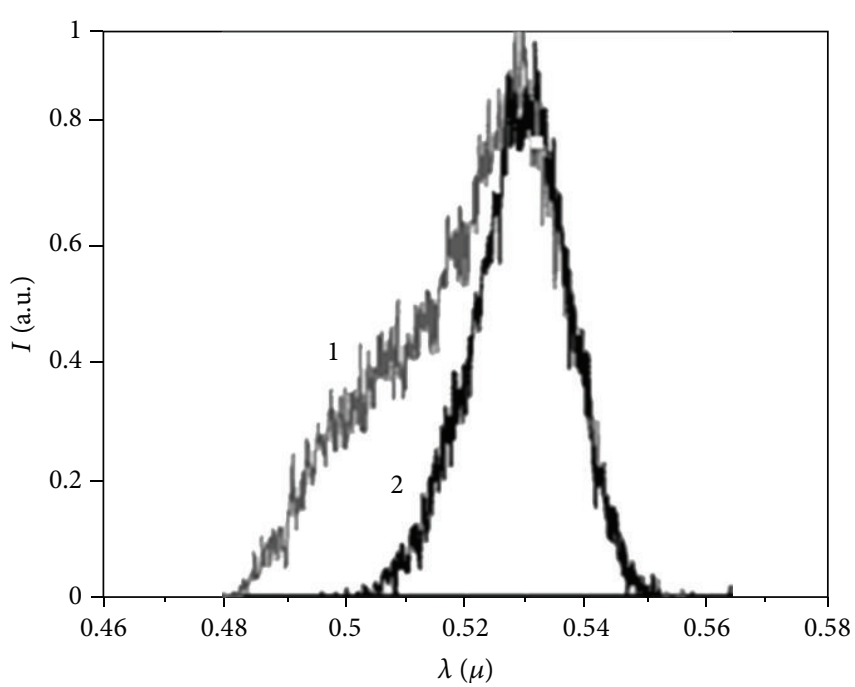

(a)

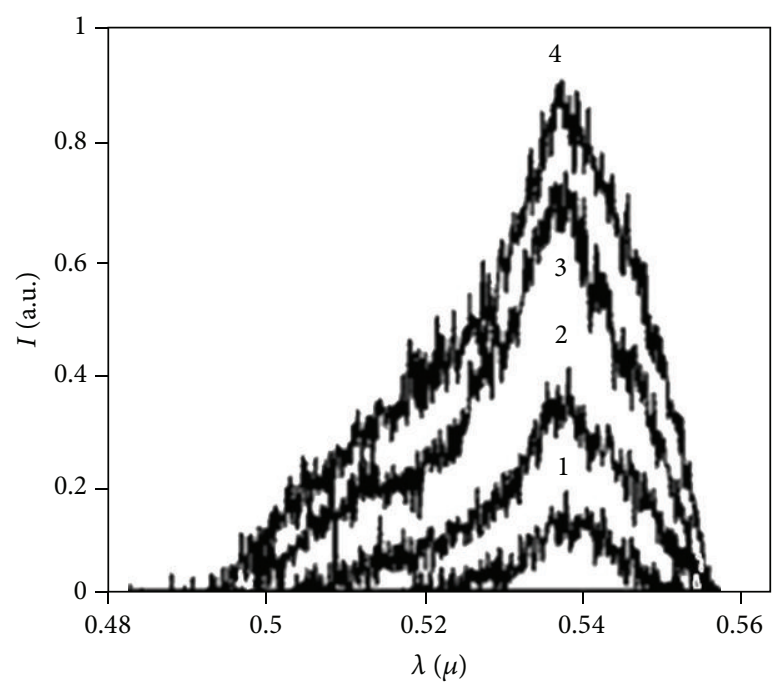

(b)

FIGURE 2: Spectra of converted broadband radiation for convergent input beams in LN: (a) type of phase matching: 1-oo-e; 2-oe-e and (b) for different temperatures of thermal object: 1-1940 K; 2-2097 K; 3-2337 K; 4-2456 K.

a spectral range of shorter wavelengths when the angle of convergence of an input beam is increased and vice versa.

In addition, when infrared radiation is focused inside a nonlinear crystal, the conversion efficiency of broadband infrared radiation is increased (Figure 3(a)). This occurs because of the increasing number of possible vector interactions at the phase matching condition for frequencies $\omega_{i}<$ $\omega_{0}\left(\omega_{i}\right.$ : frequency of input radiation). Note that with these frequencies, the phase matching condition is not fulfilled for the collinear interaction of light waves.

Moreover, the conversion efficiency of broadband infrared radiation depends on the thermal object temperature and the pump radiation parameters [9]. The increase in the thermal object temperature has very little effect on changing the experimental spectrum shape (Figure 2(b)), while the conversion efficiency is increased (Figure 3(b)). The increase in the spectral range of input infrared radiation in the region of short wavelengths results in the extension of the spectrum of the converted radiation and the increase in the conversion efficiency of broadband infrared radiation.

\section{Conclusion}

The spectra of broadband infrared radiation converted in LN crystals were calculated for convergent input beams. The calculation results show that the spectral range of converted 


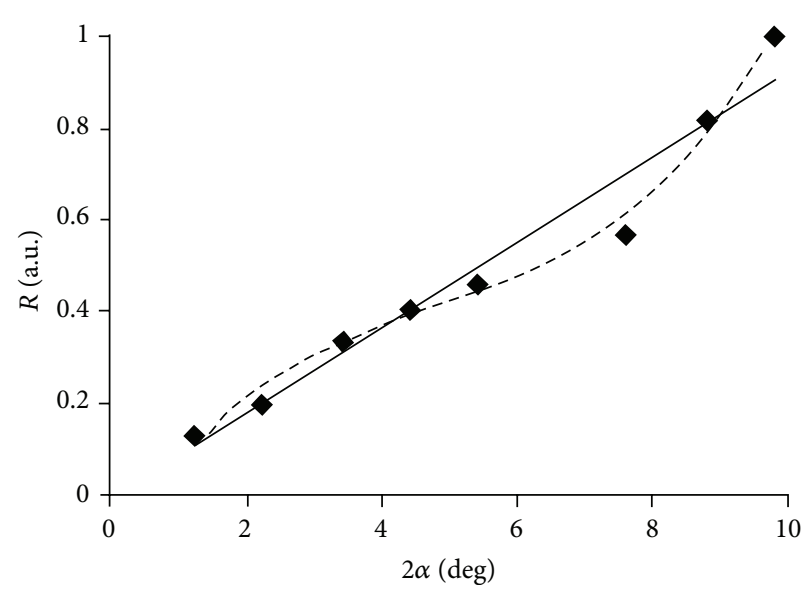

(a)

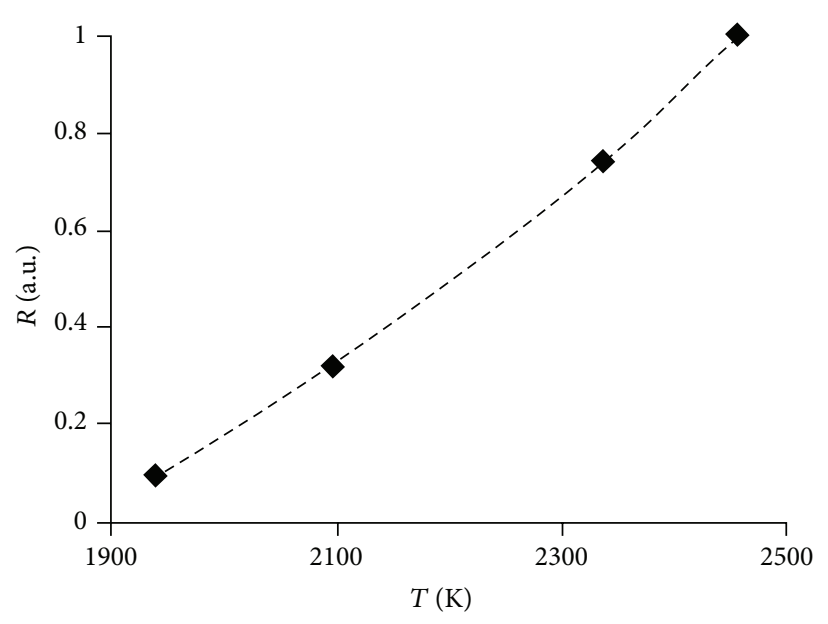

(b)

FIGURE 3: Dependence of integrated power of converted broadband radiation on (a) convergence angle of an input beams and (b) temperature of thermal object. The solid line-theoretical results, the dashed line and dots-experimental results.

radiation and the conversion efficiency depend on the convergence angle of the input beam and the pump radiation parameters such as the polarization and spectral range of broadband infrared radiation. In addition, experimental spectra were obtained under the same conditions and compared to the calculated spectra. The calculation results agree with the experimental results. The proposed algorithm for the calculation of the spectra of converted broadband radiation can be used for simulation and to study the conversion of broadband infrared radiation in different nonlinear optical crystals. The investigation results can be helpful for realizing color vision systems and for IR-object visualization.

\section{Acknowledgments}

This research has been conducted with funding from the Research Grant of Kwangwoon University in 2012-2013. The authors are grateful to Professor Vladimir I. Stroganov for the helpful discussions and for his interesting ideas. They also thank Editage for providing editorial assistance.

\section{References}

[1] M. Alfaro, G. Paez, and M. Strojnik, "Bidimensional fluorescence analysis and thermal design of europium thenoyltrifluoroacetonate based thermal-to-visible converter," Applied Optics, vol. 51, no. 7, pp. 780-788, 2012.

[2] D. Ceus, A. Tonello, L. Grossard et al., "Phase closure retrieval in an infrared-to-visible upconversion interferometer for high resolution astronomical imaging," Optics Express, vol. 19, no. 9, pp. 8616-8624, 2011.

[3] J. S. Dam, C. Pedersen, and P. Tidemand-Lichtenberg, "Theory for upconversion of incoherent images," Optics Express, vol. 20, no. 2, pp. 1475-1482, 2012.

[4] E. Bulard, M. P. Fontaine-Aupart, H. Dubost et al., "The effect of bacterial adhesion on grafted chains revealed by the noninvasive sum frequency generation spectroscopy," Spectroscopy, vol. 27, no. 5-6, pp. 571-579, 2012.
[5] J. E. Midwinter, "Image conversion from 1.6. $\mu$ to the visible in lithium niobate," Applied Physics Letters, vol. 12, no. 3, pp. 68-70, 1968.

[6] G. V. Krivoshchekov, Y. G. Kolpakov, V. I. Samarin, and V. I. Stroganov, "Conversion of broad-band light in nonlinear crystals," Journal of Applied Spectroscopy, vol. 30, no. 5, pp. 633637, 1979 .

[7] V. I. Stroganov and V. I. Troilin, "Transformation of nonmonochromatic wideband ir image in nonlinear lithium iodate and lithium formate crystals," Journal of Applied Spectroscopy, vol. 50, no. 2, pp. 222-225, 1989.

[8] V. I. Troilin and V. I. Stroganov, "Optical electronic temperature measuring system with visualization of thermal radiation," Izvestiya Vysshikh Uchebnykh Zavedenii. Priborostroenie, vol. 33, no. 5, pp. 83-85, 1990.

[9] E. V. Tolstov, V. V. Krishtop, and V. I. Stroganov, "Spectral response of a thermal-image converter," Journal of Optical Technology, vol. 71, no. 1, pp. 60-61, 2004.

[10] V. V. Krishtop, E. V. Tolstov, V. I. Stroganov, and A. V. Syui, "Converting IR radiation with broad-band pumping in nonlinear-optical crystals," Journal of Optical Technology, vol. 74, no. 4, pp. 243-245, 2007.

[11] A. I. Illarionov, O. V. Yanchuk, and A. A. Starchenko, "Effect of fundamental radiation wave front astigmatism on the process of second optical harmonic generation in uniaxial crystals," Russian Physics Journal, vol. 51, no. 11, pp. 1195-1199, 2008.

[12] V. Krishtop, V. Efremenko, M. N. Litvinova, E. Tolstov, and V. Stroganov, "The spectrum of up-converted broadband radiation in nonlinear optical crystals," in Laser Optics: Solid State Lasers and Nonlinear Frequency Conversion, vol. 6610 of Proceedings of SPIE, no. 28, 2007.

[13] M. N. Litvinova, V. I. Stroganov, E. V. Tolstov, E. D. Goncharova, and I. A. Garankova, "The conversion of broadband radiation in the nonlinear optical crystals," Pacific Science Review, vol. 12, no. 1, pp. 35-40, 2010. 

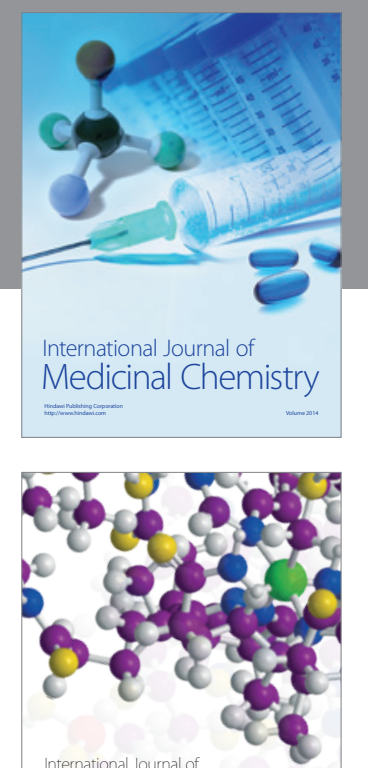

\section{Carbohydrate} Chemistry

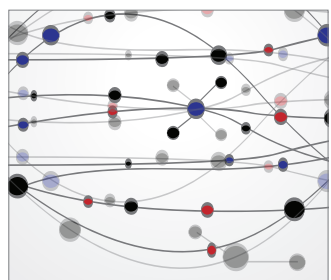

The Scientific World Journal
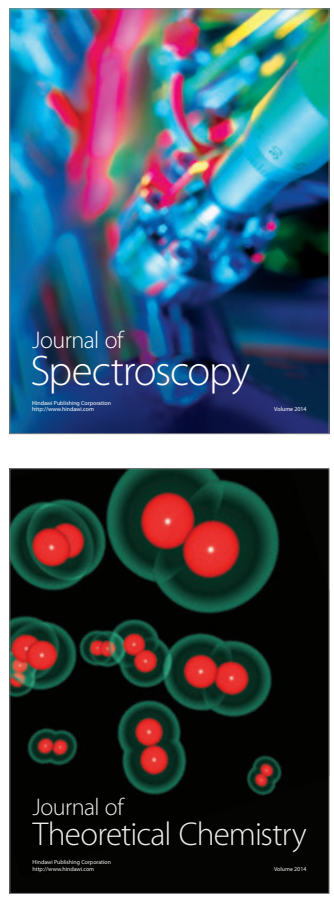
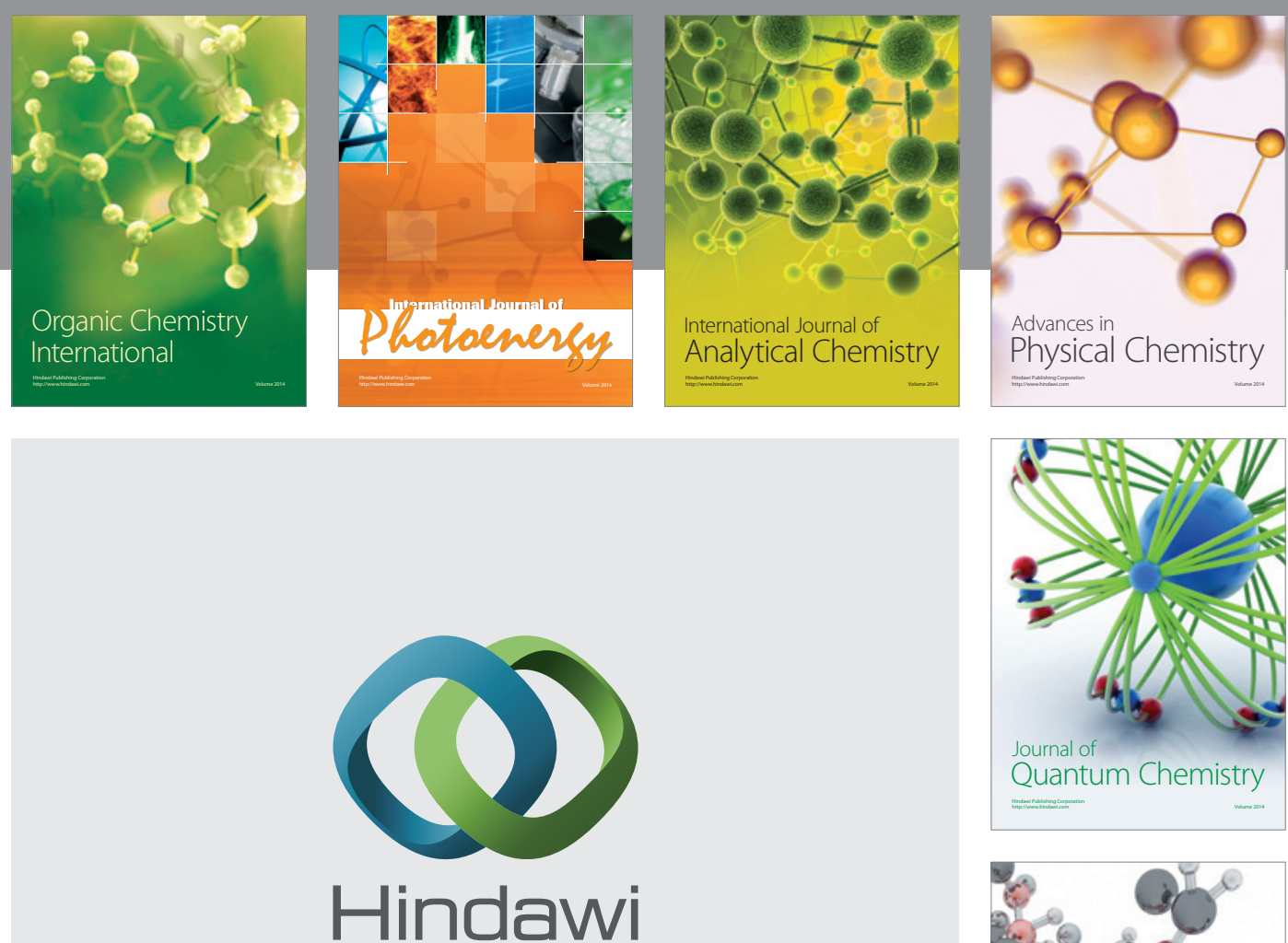

Submit your manuscripts at

http://www.hindawi.com

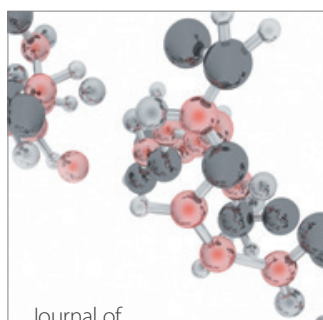

Analytical Methods

in Chemistry

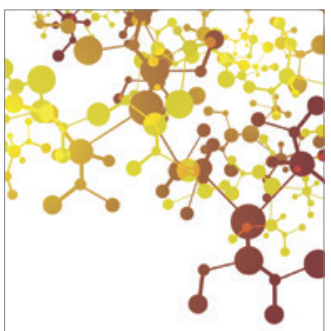

Journal of

Applied Chemistry

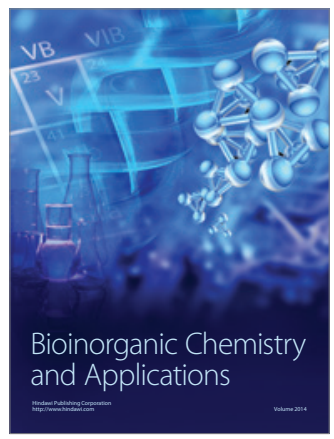

Inorganic Chemistry
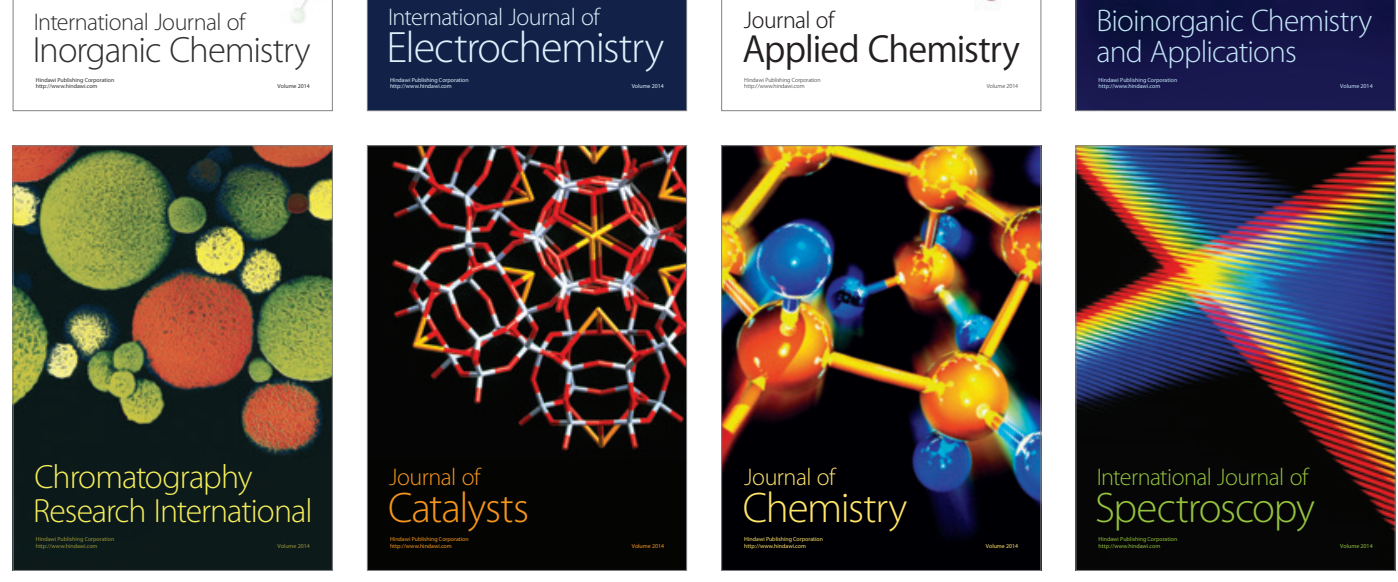